\title{
Fresh Tea Picking Robot Based on DSP
}

\author{
Heng $\mathrm{Li}^{1}$, Chao $\mathrm{Li}^{1}$, Liming $\mathrm{Xu}^{1}$, Guangming Qin ${ }^{2}, \mathrm{Xin} \mathrm{Lu}^{1}$, and Ying Zhao ${ }^{2}$ \\ ${ }^{1}$ College of Engineering, China Agricultural University, QingHua East Road No.17, \\ Beijing, China \\ ${ }^{2}$ Nanjing Agricultural Machinery Research Institute, Nanjing 210014 \\ \{liheng06,xlmoffice\}@126.com, caulc2012@163.com
}

\begin{abstract}
According to the tea cultivation conditions a 4 DOF gantry Cartesian coordinate tea-picking robot was designed.The picking gripper was used two fingers, droven by a servo to open and close. One finger was furnished a knife to cut the tea steer. The controlling system was based on TMS320F2812 DSP and was consisted of both $\mathrm{C}$ and assembly language. It was able to achieve serial communication, data processing and precise servo control. The tea picking robot work efficiency verification tests and linkage comparative test have been conducted in the indoor. The results show that the maximum operating frequency of motor drive system of $\mathrm{X}$-axis, $\mathrm{Y}$-axis and $\mathrm{Z}$-axis were $173.61 \mathrm{kHz}, 58.59 \mathrm{kHzand}$ $24.40 \mathrm{kHz}$ respectively, and that two-axis linkage and three-axis linkage operation efficiency were 1957 times per hour and2517times per hour respectively.
\end{abstract}

Keywords: tea, picking robot, DSP, servo control, two-axis and three-axis linkage.

\section{Introduction}

The two main ways to pick tea are artificial picking and mechanical harvesting. But the artificial picking is labor intensive and highly cost, and the mechanical picking uses the method of cutting, so it has a high breakage rate and is harm to the tea trees. Also it makes the subsequent sorting more difficult and it is not suitable for picking famous tea. Robot application in this field in China or abroad is still in the blank. This paper developed a TMS320F2812 DSP controlled 4 DOF tea-picking robot which can pick various kinds of tea piece by piece.DSP is a high-performance microprocessor in digital signal processing and controlling, which has the character of high speed, high efficiency, strong anti-jamming capability and strong scalability. It can meet the high-speed and real-time demand.

\section{The Picking Robot Mechanism Design}

\subsection{Tea Cultivation}

In order to meet the needs of the job of the tea-picking robot, tea trees were specially cultivatated.The tea garden space diagram was showed in Fig. 1. The ridge width of tea trees is $1.2 \mathrm{~m}$, the height is between $0.5 \mathrm{~m}$ and $0.8 \mathrm{~m}$, and the row space is $1.5 \mathrm{~m}$. The robot works across in the tea ridge and goes forward with the caterpillar tractor. 


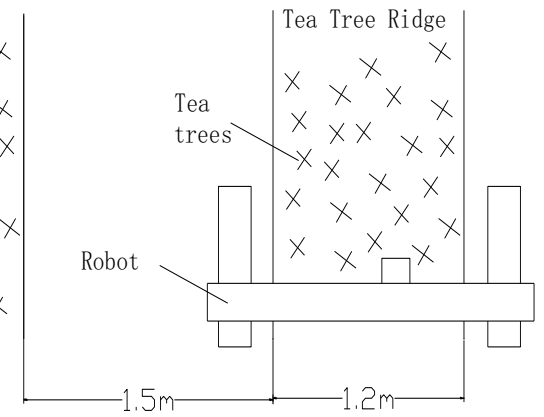

Fig. 1. The tea garden space diagram

\subsection{Design of the Tea Picking Robot Mechanism}

According to the dimensions of the tea ridge, a 4 DOF gantry Cartesian coordinate tea-picking robot was designed. The robot manipulator (Fig. 2 (a)) consisted of X1 slide, X2 slide, Y slide, Z slide, picking gripper, synchronous belt, synchronous belt pulley and some connecting plates. The effective stroke of $\mathrm{X}$-axis, $\mathrm{Y}$-axis and $\mathrm{Z}$-axis are $500 \mathrm{~mm}, 1300 \mathrm{~mm}$, and $200 \mathrm{~mm}$ respectively. The $\mathrm{Y}$-axis is connected across the two $\mathrm{X}$-axis and the $\mathrm{Z}$-axis is installed on the $\mathrm{Y}$-axis. In order to ensure that X1-axis and $\mathrm{X} 2$-axis move synchronously the two axes are connected by a synchronous belt.

According to the specific requirements of the tea picking, a steering engine driven picking gripper was designed (Fig. 2 (b)), which was installed on Z-axis. The left gear of the gripper was connected and rotate with the rotating shaft of the steering engine. The right gear achieves the opposite direction of rotation through a gear pair with the left gear, and together with the four bar linkage mechanism $(18,19,24,25)$ achieving the opening and closing of the gripper. When the blade and the blade pad installed on the front end of the two fingers meshing together,the tea can be cut down.

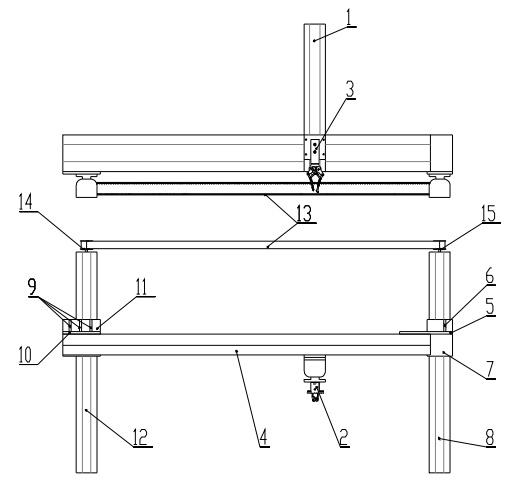

(a) Manipulator

Fig. 2. Tea Picking Robot mechanism 


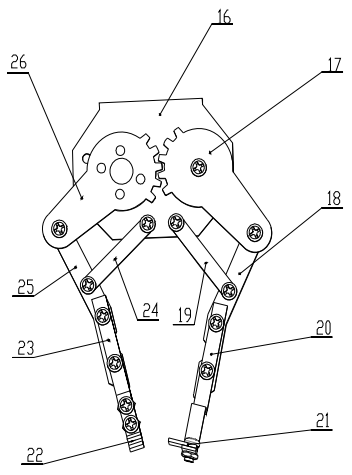

(b) Picking Gripper

1-Z slide;2-Picking gripper;3-Gripper fixed plate;4-Y slide;5-Right vertical mounting plate of $\mathrm{Y}$ slide;6-Right rib of $\mathrm{Y}$ slide;7-Right horizontal mounting plate;8-X2 slide(driven);9-Left rib of $\mathrm{Y}$ slide;10-Left vertical mounting plate of $\mathrm{Y}$ slide;11-Left horizontal mounting plate of $\mathrm{Y}$ slide;12-X1 slide(driving);13-Synchronous belt;14-Left synchronous pulley;15-Right synchronous pulley;16-Gripper bracket;17-Right gear of gripper;18-Right finger;19-Right connecting rod;20-Blade bracket;21-Blade;22-Blade pad;23-Bracker of blade pad;24-Left connecting rod;25-Left finger;26-Left gear of gripper(driving)

Fig. 2. (Continued.)

\section{The Control System of the Tea Picking Robot}

\subsection{Hardware Components and Principle of DSP Control Board}

The TMS320F2812 chip from TI company was selected to be the control chip. This chip is a 32-bit fixed-point DSP chip which is suitable for use in industrial control, motor control etc. It's running clock can reach $150 \mathrm{MHz}$, and each instruction cycle is $6.67 \mathrm{~ns}$. It has $128 \mathrm{k} \times 16$-bit on-chip FLASH, $18 \mathrm{k} \times 16$-bit SRAM and abundant peripheral interfaces. In order to reduce the difficulty of the system design, the mature development board QQ2812 was selected. This development board equipped with the F2812 chip and commonly used peripherals and interfaces. Fig. 3 shows the system function block diagram. The communication between the image processing unit and the DSP controller was achieved by RS232 serial port, transmitting the three-dimensional coordinate information of each plucking. According to the position gotten from image processing unit, the DSP can achieve the close-loop control of DC servo motor on $\mathrm{X}, \mathrm{Y}$ and $\mathrm{Z}$ axis and the open-loop control of gripper steer engine. 


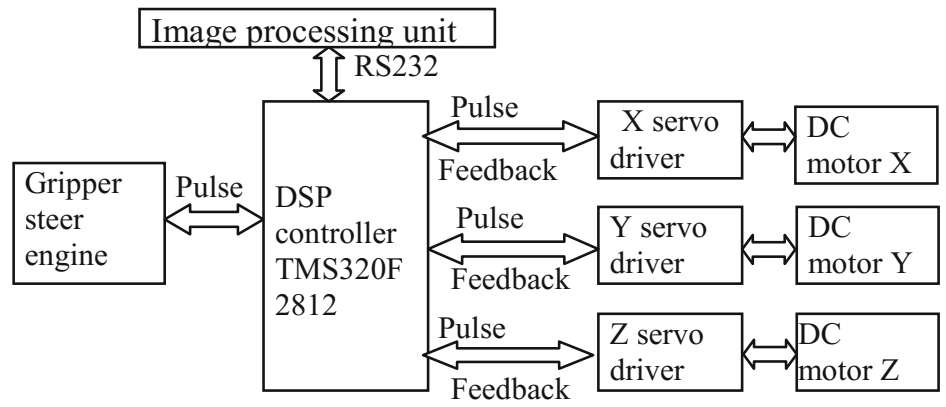

Fig. 3. System block diagram

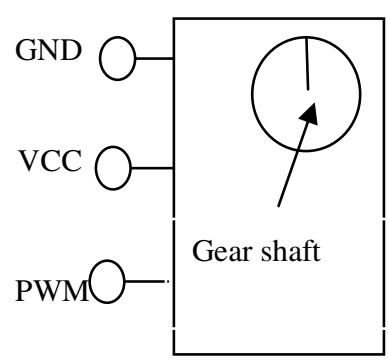

Fig. 4. Analog servo

\subsection{Servo Control System}

The structure of the analog servo was showed in Fig. 4, which was used in this system. The servo control signal is PWM signal, changing the duty cycle can change the angular position of the gear shaft of the servo. The DSP output two different duty cycle of the pulse signal corresponding to the gripper opening and closing state.

\subsection{Motor Control System}

This system has 3 DC servo motor whose model is MAX32. Due to the system's mobile power and take energy issues into consideration, a small probability $20 \mathrm{~W}$ DC motor and dear reducer drive program was selected. After the DSP receive the txt data file from image processing unit, the data was specially processed and convert into the position information of the respective axes. Then DSP sent a certain number of pulse to control the motor to rotate a certain angle, so that the each axis can move to the specified location[4,5]. The control flow diagram of the servo motor was showed in Fig. 5. The motor control system was composed of three control loops which are current loop, velocity loop and position loop. Under the work condition each motor moves according to DSP's construction, and through the three closed loop DSP can achieve high precision velocity control and position control[5,6]. 


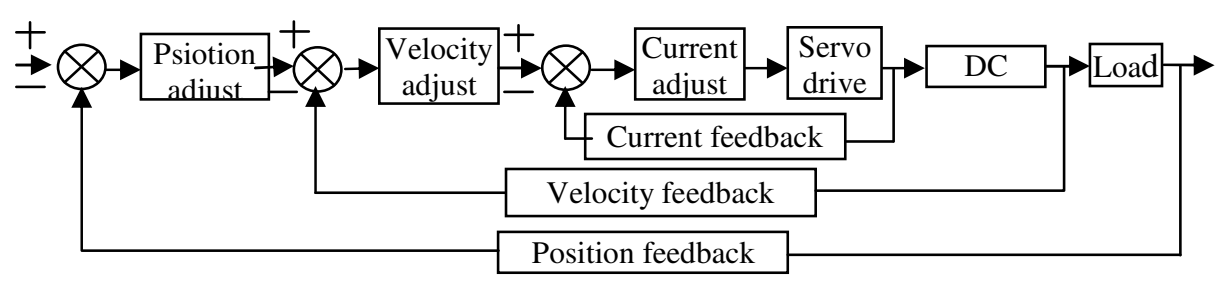

Fig. 5. Motor control block diagram

\subsection{Software Design}

The software was designed by CCS3.3(Code Composer Studio), using C language and assembly language to achieve all the function. The workflow was showed in Fig. 6. System is initialized after powered on, then steps into the communicating stage, receiving position data from the image processing unit, after which $\mathrm{X}, \mathrm{Y}$ and $\mathrm{Z}$ axis move and the gripper close, picking the tea. Simultaneously, the blower work and the tea is drawn back into the collection box, completing a picking action. Then the system will detect whether all the position have been completed. It will go to next area ,if completed. Otherwise, it will go to the next position. After having been developed and debugged, the software was flashed into the FLASH memory of the DSP from the JTAG port via USB emulator[7,8,9]. Then it was copied to RAM when working to improve the running speed. After powered on, the system orderly calls concerning functions which contain initializing function, position initializing function - pos_init(), communicating function-data_read(), coordinate detecting fuction-pos_dect(), 5 action functions and 3 DSP counter overflow interrupt functions. Table 1 shows the main function and function description.

Table 1. Key functions and use

\begin{tabular}{lll}
\hline Function name & characteristic & Capability \\
\hline Initialization() & Initializating function & Initializing concerned register \\
pos_init() & Task function & Each axis goes to original point \\
data_read() & Task function & Serial communication \\
pos_dect() & Task function & Detectingposition exceed the maximum \\
& & stroke or not \\
move_xy(m,n) & Task function & X,Y move \\
Z_down(m) & Task function & Z axis goes downward \\
paw_close() & Task function & Gripper closes \\
z_up() & Task function & Z axis goes upward \\
paw_open() & Task function & Gripper open \\
eva_t1pint_isr() & Interrupt function & X-axis position interrupt respond \\
eva_t2pint_isr() & Interrupt function & Y-axis position interrupt respond \\
evb_t3pint_isr() & Interrupt function & Z-axis position interrupt respond \\
\hline
\end{tabular}




\subsection{Communication}

This system uses standard RS232 interface as a means of communication(Fig. 7). After initializating, the DSP enter the serial communication routines waiting for the data stransmition from image processing unit. The DSP needs 4 bits decimal coordinates information, so a special processing method was used, which filters the received data and remains the thecharacters whose ASSIC value is between '0'to '9', storing the valid chatacters in a structure in the format of every 4 characters a group as a 4-bit integer and using a particular 4-bit digits as a sign of the end of the data.

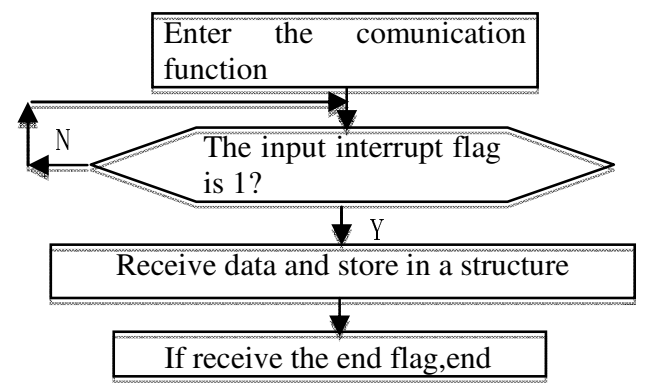

Fig. 6. Flowchart of serial communication

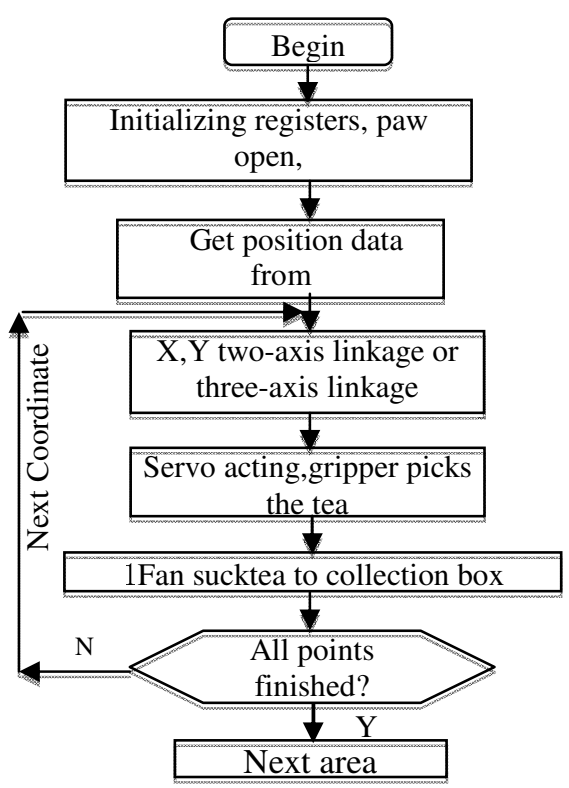

Fig. 7. Software flow chart 


\section{The Efficiency Analysis and Test of the Tea Picking Robot}

\subsection{The Efficiency Principle Analysis of the Tea Picking Robot}

The robot is showed in Fig. 8, which is consisted of control board, X silde, Y slide, Z slide, gripper etc. It is install on a crawler tractor when working. The picking speed of the robot detemines the final operating effciency which is influenced by to factors, the software factor and the hardware factor.

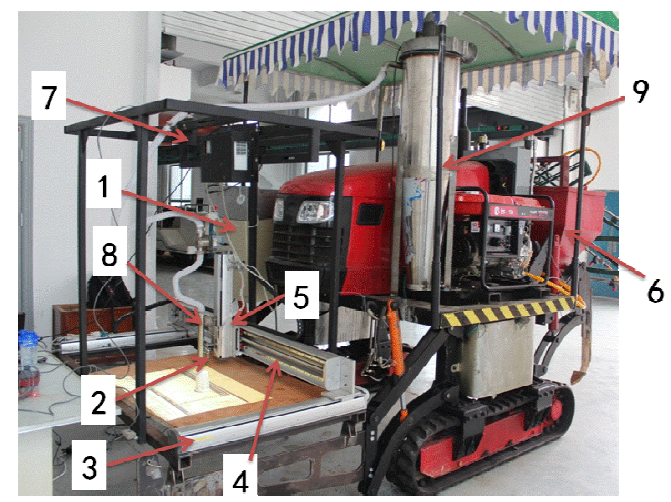

1-control board, 2-gripper,3-X slide,4-Y slide,5-Z slide,6-crawler tractor,7-camero 8 -suction tube 9-collecting box

Fig. 8. The whole system

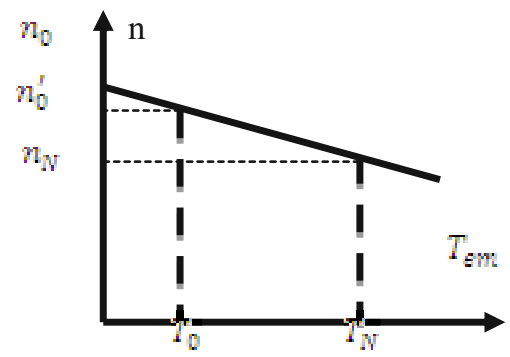

$\mathrm{n}_{0}$-Ideal no load speed ; $\mathrm{n}_{0}^{\prime}$-Actual load speed ; $\mathrm{n}_{\mathrm{N}}$-Rated speed; $\mathrm{T}_{0}$-No load torque $; \mathrm{T}_{\mathrm{N}}$-Rated torque ; Tem-Load torque

Fig. 9. Mechanical characteristic curve of DC motor 


\subsection{Software Factor}

The TMS320F2812 DSP has two event managers, each of which has two independent counter. Adjusting the upper limit value can change the overflow cycle, so as to achieve the purpose of changing the pulse frequency. Formular (1) is the output pulse frequency formular $[9,10]$.

$$
\mathrm{f}=\frac{H S P C L K}{(T X P R+1) 2^{T P S}}
$$

f-output frequency; TxPR-overflow value of counter(x is 1,2,3 or 4); TPS-division factor of counter; HSPCLK-system clock pulse frequency.

\subsection{Hardware Factor}

The physical characteristics of the motor is another factor affecting the effciency. Formular (2) is the DC motor speed formular and the mechanical characteristic curve of DC motor is showed in Fig. 9 [5].

$$
\mathrm{n}=\frac{U}{\emptyset C_{e}}-\frac{R}{\emptyset^{2} C_{e} C_{T}} T_{e m}=n_{0}-\beta T_{e m}
$$

$n_{0}$-Ideal no load speed $; \beta$-Equivalent load curve slope ;Tem-Load torque ;U-Rated voltage ; $\varnothing$-Excitation flux ; R-Armature resistance ; $C_{e}$-EMF constant; $C_{T}$-Asynchronous motor torque constant.

\subsection{Test of the Tea Picking Robot}

\subsubsection{Maximum Working Test of the Motor System}

1) Test purpose. In order to test the actual hightest working speed, a operating frequency test was done.

2) Test method. When not connected to the inage processing unit, the hightest working speed of each motor system were tested by adjusting the ouput pulse frequency through the CCS software. The output frequency was changed by changing the value of TXPT in the initializing function. The word length of the DSP is 32 bits, and it's initialization value of the register is consited of 4 hexadecimal numbers whose format is as following:

$$
\text { TXPR }=0 \times 1234
$$

Aiming to test the minmum value of TXPR(corresponding to the maximum value of the output pulse frequency), change the second bit of the right number from the right to the left successively and compile and debug the coftware online, and then test whether the motor system can work normally[10].

3) Test results. The frequency test histogram obtained through 3 times of repeated tests was showed in Fig. 10. The test value of $\mathrm{X}$-axis for each time were $173.53 \mathrm{kHz}, 173.69 \mathrm{kHz}$ and $173.62 \mathrm{kHz}$ respectively. Correspondingly, the value of $\mathrm{Y}$-axis were $58.61 \mathrm{kHz}, 58.50 \mathrm{kHz}$ and $58.57 \mathrm{kHz}$ respectively, the value of $\mathrm{Z}$-axis were $24.38 \mathrm{kHz}, 24.44 \mathrm{kHz}$ and $24.51 \mathrm{kHz}$ respectively. Finally, the average hightest frequency of $\mathrm{X}, \mathrm{Y}$ and $\mathrm{Z}$ axis were $173.61 \mathrm{kHz}, 58.59 \mathrm{kHz}$ and $24.40 \mathrm{kHz}$ respectively. 


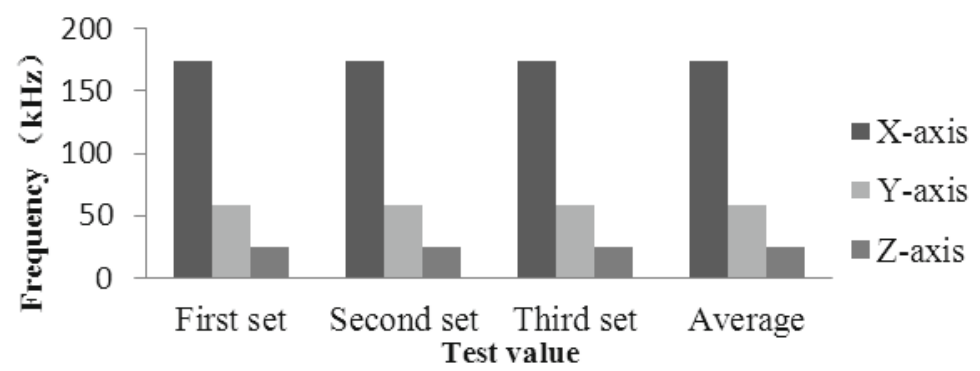

Fig. 10. Frequency test

4)Analysis. Because only the second bit of TxPR was changed in the initializing function and the lowest bit was not, there was a test error which was within $\pm 0.1 \mathrm{KHz}$ according to Formular (1). Analyzed from the Formular (1) and Fig. 9, the system has the following factor impacting the maximum operating frequency.

(1) The actual no load speed of the motor- $n_{0}^{\prime}$. The bigger the $n_{0}^{\prime}$ is the fast the motor can rotate.

(2) System damping, containing the friction damping of the ball screw, the friction damping the linear track, the friction damping of the motor and the redactor. The increase of the system damping can cause the increase of the load torque, thereby causing the speed drop.

(3) Load. The increase of the load would cause the speed drop also.

(4) The reduction ratio. Under a fixed load torque the bigger the reduction ratio of the redactor is the faster the motor can rotate, which is to say the output pulse frequency can be higher.

\subsection{Linkage operation Effectiveness Test}

\subsubsection{Test Purpose}

Aiming to compare the operation efficiency and the advantages and disadvantages between the two-axis and three-axis linkage, the efficiency test was done.

\subsubsection{Test Method}

Here the avareage picking time every time was tested under different linkage. Because the distance between each tea leaf are roughly eaque, so the coordinate point spcaing was artificially arranged at equal intervals, 20mm. Table 2 shows the coordinate parameter.

Table 2. Coordinate parameter (Unit: $\mathrm{mm}$ )

\begin{tabular}{|c|c|c|c|c|c|c|c|c|c|c|}
\hline (2014in & Poin & Point 2 & Point 3 & Point 4 & 4 Point 5 & 5 Point 6 & Point I & $\mathrm{PO}$ & 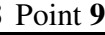 & Poin \\
\hline $\mathbf{X}$ & 20 & 40 & 60 & 80 & 100 & 120 & 140 & 160 & 180 & 200 \\
\hline $\mathbf{Y}$ & 20 & 40 & 60 & 80 & 100 & 120 & 140 & 160 & 180 & 200 \\
\hline $\mathbf{Z}$ & 30 & 30 & 30 & 30 & 30 & 30 & 30 & 30 & 30 & 30 \\
\hline
\end{tabular}




\subsubsection{Experimental Results and Analysis}

The test was repeated three times to test the 10-point overall time. The experimental results were showed in Fig. 11. In the first set of test, the two-axis linkage total time was 18.41 seconds, and the three-axis linkage is 14.24 seconds. The second set of test results are 18.56 seconds and 14.37 seconds. The third set of test resluts are 18.32 seconds and 144.40 secondsrespectively. By calcultating the average value of overall time and the average time per time, the average picking time each time of two-axis and three-axis linkage are 1.84 seconds and 1.43 seconds respectively. Then the efficiency of each linkage can be determined.The two-axis sequence of operation is that $\mathrm{X}, \mathrm{Y}$ axis move simultaneously, when arriveing the destination, the $X$ axis moves, and after $Z$ reaches the special location the gripper acts, then the $\mathrm{Z}$ axis goes back to original position. Characteristics of two-axis linkage is that the gripper moves up and down verticaly which will not collide with the surronding tea trees, but has a slower speed and relatively lower effciency. The three-axis sequence of operation is that $\mathrm{X}, \mathrm{Y}$ and $\mathrm{Z}$ axis move simultaneously, when arriveing the destination gripper acts, then $\mathrm{X}, \mathrm{Y}$ and $\mathrm{Z}$ axis go to next position. Characteristics of three-axis linkage is that it has a higher speed, but may collide with the surronding tea trees. Three-axis reduces the independent operation time of Z-axis, and the data obtained from experiments shows that these two action took 0.41seconds.According to calculating the work frequency hourly, the two-axis linkage is 1957 times per hour and the three-axis linkage was 2517 times per hour, so the three-axis linkage has a obvious speed advantage.

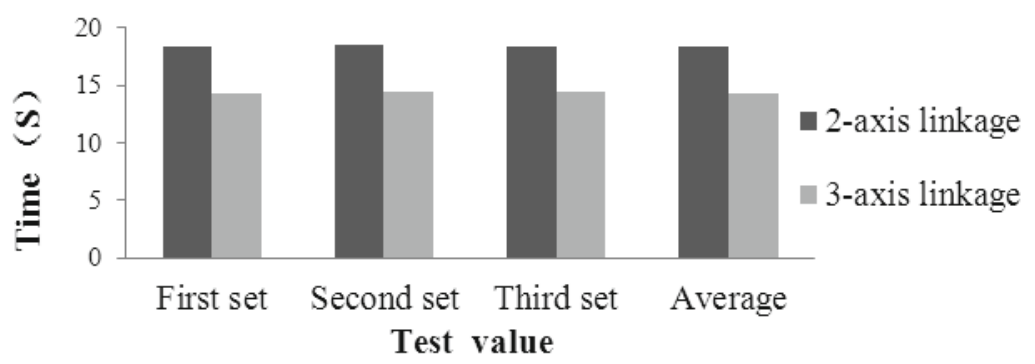

Fig. 11. Linked experimental result

\section{Conclusions}

Based on the tea cultivation conditions a TMS320F2812 DSP controled 4 DOF tea-picking robot was disigned. Based on the experimental verification, the following conclusions were drawn:

1)A 4 DOF gantry Cartesian coordinate tea-picking robot was designed and a gripper who can pick and cut was designed. This robot can pick tea across the ridge whose structure could meet the basic operational requirements.

2)The high-performance TMS320F2812 DSP can achieve the real-time control, rapid response, and fast communication. It also can convert the coordinate positions into the number of pulses accurately, achieve the real-time interrupt response accurately, position the gripper accurately and achieve the close loop control of the robot. 
3)The theoretical analysis of the working efficiency was finished in the indoor test, and the following factor saffecting the working efficiency were fund: the output pulse frequency, the ideal no load speed, the system damping, the load, the gear ratio of the redactor and the linkage form. The actual working frequency of the motor system was obtained through the frequency test which were $173.61 \mathrm{kHz}, 58.59 \mathrm{kHz}$ and $24.40 \mathrm{kHz}$ respectively. The linkage comparison test indicated that the operating sequence and linkage formate can be changed by changing the parameters of the control software, that the efficiency of two-axis linkage and three-axis linkage were 1967 times per hour and 2517 times per hour respectively.

4)The robot was only tested indoor, also need test in the field in the future.

Acknowledgements. This project was a "National scientific support project" in rural areas (2011BAD020B07-3) which was financially supported by the Department of Science and Technology of China. The Key Technology and Equipment Research and Development of Tea Work Robot (BE2011345).

We gratefully acknowledge the help and support providers from The Ministry of Agriculture Nanjing Institute of agricultural mechanization, Yangzhou University and Nanjing Forestry University.

\section{References}

[1] Wang, Y., Chen, A., Liu, X.: The rectangular coordinate robot control system. Light industry machinery, Ning Du Yunsheng high-tech research institute (fourth period 2010)

[2] Kuwahara, J.H., Ono, Y., Nikaido, M., Matsumoto, T.: A Preceion Direct-Drive Robot ARM. Yokogawa Hokushin Electric Corporation, Corporate R \& D, Musashino-shi

[3] Zhou, G., Li, Z., Jiang, F.: DSP and CPLD-based open robot controller. Control Engeineering, 84-86 (first period, 2006)

[4] Lei, A.: Robot motion control software design. Master thesis, Southeast China University (2008)

[5] Chen, L., Yan, Z., Liu, X.: Control Motors, 3rd edn. Xi' an Electronic and Science University Press, Xi' an (2000)

[6] Zhang, B., Zhao, H., Yue, Y.: DSP-based stepper motor control system design. Instrument Technique and Sensor (8), 63-66 (2010)

[7] Xu, X., Yu, J., Zhang, L.: Stepper motor multi-axisthe DSP control system design. Mechanical and Electrical Product Development and Innovation 18(5), 110-112 (2006)

[8] Tang, M.: The develop of ARM-based programmable controller. Nanjing University of Information Engineering, Nanjing (2007)

[9] Wang, L., Zhang, S., Ma, C., Xu, Y., Qi, J.: ARM -based variable spraying control system design. Jilin University Key Laboratory of the Ministry of education (2010)

[10] Ren, R., Zhou, L., Yao, G.: TMS320F2812 Source code analysis. Publishing House of electronics industry, China (2012) 